\title{
Hierarchical Region Based Template Matching Technique for Global Motion Reduction of Coronary Cineangiograms
}

\author{
K. A. S. H. Kulathilake, L. Ranathunga, G. R. Constantine, and N. A. Abdullah
}

\begin{abstract}
The Coronary Cineangiogram (CCA) is an invasive medical image modality which is used to determine the stenosis in the Coronary Arteries. The global motion occurring due to the heart beat makes great disturbance to obtain the visual alignment among the vessel structure shown in the CCA frames. Therefore, the recorded vessel structure's position in CCA varies within the frame sequence. This paper describes a hierarchical region based template matching technique to reconstruct the $\mathrm{CCA}$ by reducing the global motion artifacts. This proposed motion reduction technique is efficient and it reconstructs the CCA by reducing the background motion as desired. Experimental results of this method have shown its' ability to maintain the visual alignment of the internal blood flow among the frames.
\end{abstract}

Index Terms-Angiography, computer aided diagnosis, image motion analysis, medical diagnostic imaging component.

\section{INTRODUCTION}

Coronary Angiogram is one of the invasive medical image modalities used by the clinical practitioners as a preliminary diagnostic technique to detect luminal obstruction or the degree of stenosis. Coronary Angiograms are produced either as still images or as videos (Cineangiogram) [1]. The diagnosis based on Coronary Angiogram is subjective because, the results are produced through the visual judgments by considering the contrast agent flow. Hence, in most cases, these assessments fail due to the visual hindrance and lack of quantification capabilities not only in image based Coronary Angiograms but also Coronary Cineangiograms (CCA) [2], [3].

It is desirable to do an objective assessment of detected stenosis based on the functional significance of Coronary Arteries such as flow rate and flow velocity recorded in the CCA [1], [4]. However, the motion artifact occurs due to the heart beat (global motion) and patient movement makes some visual disturbances to do the objective assessments using CCA. Further, it is difficult to envisage the visual alignment among the vessel structure recorded in the frame sequence of CCA because, the position of the vessel structure varies

Manuscript received March 18, 2014; revised May 14, 2014. This investigation received financial support from the National Science Foundation, Sri Lanka under Grant No. NSF/SCH/2013/06.

K. A. S. H. Kulathilake and L. Ranathunga are with the Department of Information Technology, University of Moratuwa, Moratuwa, Sri Lanka (e-mail: hemanthak@uom.lk, lochandaka@uom.lk).

G. R. Constantine is with the Department of Clinical Medicine, Faculty of Medicine, P.O. Box 271, No 25, Kynsey Road, Colombo 08, Sri Lanka (e-mail: godwinconstantine@live.com).

N. A. Abdullah is with the Department of Computer System and Technology, Faculty of Computer Science \& Information Technology Building, University of Malaya, 50603 Kuala Lumpur, Malaysia (e-mail: noraniza@um.edu.my). within the sequence of frames in CCA due to the motion artifacts [5] (see Fig. 1).

Therefore, we aim to develop a mechanism that can reduce the motion artifact in the CCA to obtain visual alignment of the vessel structures among the CCA frames. Our proposed method introduces a Hierarchical Region based Template Matching (HRTM) technique to reduce the global motion from the CCA to maintain the visual alignment among the Coronary Arteries shown in the frames.

The rest of the paper is organized as follows: The recent research findings related to the motion compensation of Angiography are explained in Section II. The methodology of the proposed technique is described in Section III. Experimental results of the method are reported in Section IV, followed by discussion on the results. Finally, the conclusion section briefly explains the future work that can be extended using the results of this proposed technique.

\section{BACKGROUND}

This section briefly reviews some selected recent publications which explain some research attempts to estimate and eliminate the motion artifacts from Coronary Angiography. Scale Invariant Feature Transform (SIFT) based motion estimation and video stabilization technique was explained in [5]. In this study, the SIFT was used to estimate the translational distance and direction between two frames and the motion compensated video was created using those estimations.

Image registration based motion compensation in Angiography has been reported in many research publications. Kumar and team has explained the application of image registration techniques for motion correction in Digital Subtraction Angiograms [6]. As the image registration techniques; a light weight modified demons method and a constraint-based inverse consistence image registration method has been applied. Meijering et al. have also applied the image registration technique for Coronary Angiograms to produce motion compensated Angiograms effectively and efficiently [7]. Ko, Mao and Sun have introduced a multiresolution based image registration approach and their method automatically registers the arterial structures in the areas of interest selected from a pair of sequential images [8]. Further, it provides a sub pixel precision in registration [8]. Reference [9] discusses about an intensity based medical image registration tool box which consists of a collection of algorithms that are commonly used to solve medical image registration problems. Meunier and team proposed optical flow based method to compute the regional epicardial deformation from CCAs [10]. The proposed algorithm can track the motion of the Coronary 
Arteries as a whole and quantify the two dimensional deformation of the epicardial surface locally. Zheng and
Weirong also analyzed the Arterial dynamics using an optical flow based technique and elastic registration [11].
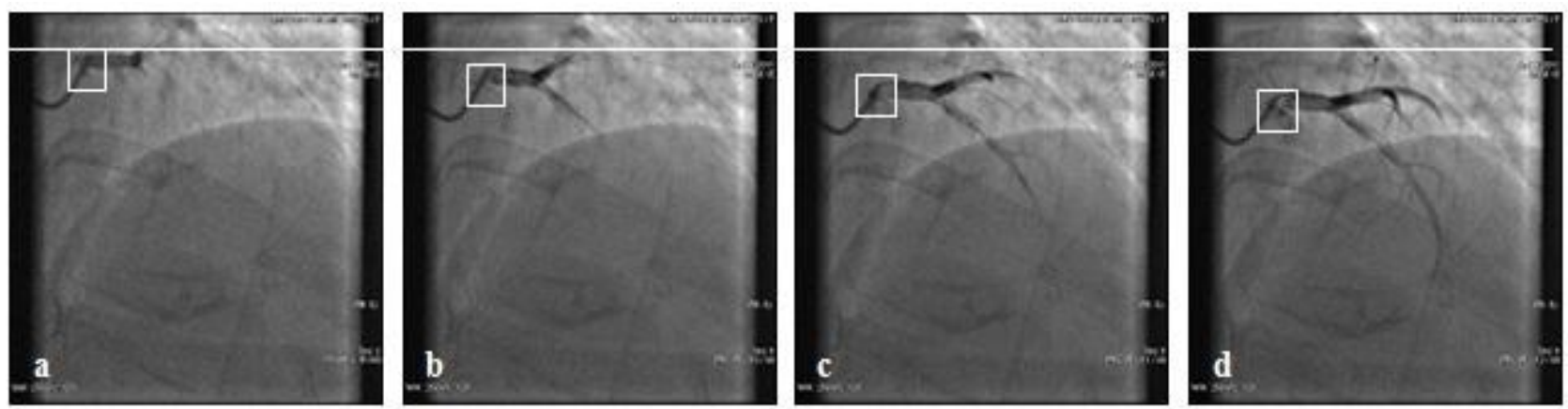

Fig. 1. Motion artifacts in CCA; Catheter engaged area is marked using whit colored square and the placement of it varies in the consecutive frames shown in a,b,c and d, as a result of the global motion. The distribution of contrast agent is gradually increasing in all images and it is denoted as local motion.

\section{Methodology}

In order to achieve the vessel alignment within the frame sequence of CCA, the global motion must be calculated and reduced from each frame of the CCA. There are mainly four steps available in our proposed HRTM motion reduction technique: preprocessing, motion estimation, frame alignment and motion eliminated video creation. An original CCA of frame rate $15 \mathrm{fps}$ is input to the proposed method and it produces a global motion compensated CCA as the output.

\section{A. Preprocessing}

It was revealed that the CCA frames consist of noise and nonuniform illumination problem [3], [5]. As a result of that, the visual quality of the recorded CCA is degraded. Further, the illumination variations provide some incorrect results in template matching procedures. Therefore, the objective of the preprocessing stage is to apply possible image enhancement techniques to obtain the required visual quality of the vessel structures recorded in CCAs.

A median filter with kernel size $3 \times 3$ was applied to the CCA frames as a noise removal technique. Afterwards a homomorphic filter was applied to extract the nonuniform illumination component of the CCA [12]. An image $(P)$ with spatial coordinates $(x, y)$ consists of illumination $(i)$ and reflectance $(r)$ components and it can be expressed by taking the natural logarithmic as follows;

$$
P(x, y)=\ln \{i(x, y)\}+\ln \{r(x, y)\}
$$

We applied the Discrete Fourier Transform (DFT) to the logarithmic image and as a result of that, the illumination components of the image can be easily identified through the low frequency content in the frequency domain because the illumination is considered as a slowly varying pattern in an image. Afterwards, the butterworth low pass filter was applied to extract the low frequency components from the frame to be processed and the transfer function of the butterworth low pass filter is given in (2);

$$
S(u, v)=\frac{1}{1+\left[D(u, v) / D_{0}\right]^{2 n}}
$$

where, $S(u, v)$ is the result image, $D_{0}$ is the distance from origin to obtain the cutoff frequency, $D(u, v)$ is the radial distance from the origin and $n$ is the order. After the filtering, inverse DFT has been applied to transform the image from frequency domain to the spatial domain. Then the illuminated component is extracted from the processed image to obtain the uniformly illuminated image.

The processed image is normalized to obtain the best quality contrast among the vessel structures shown in each frame. Fig. 2 depicts the original CCA frame and the respective preprocessed CCA frame for further clarification.

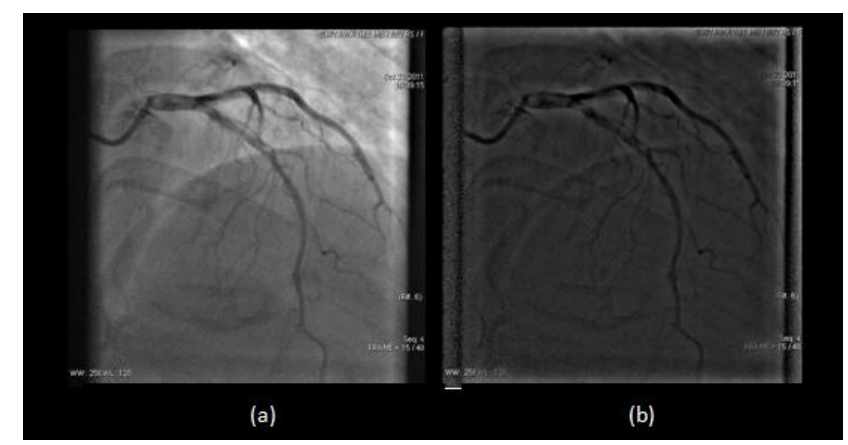

Fig. 2. Preprocessing (a) Original CCA frame with nonuniform illumination (b) Uniformly illuminated CCA frame

\section{B. Motion Estimation}

Motion estimation process of the proposed method is based on template matching image processing technique and mainly consists of two phases namely; (i) Template selection and (ii) applying HRTM. Following section explains those two phases briefly.

Template selection is done as an interactive activity using the first frame of the input CCA to be processed. The user can arbitrarily select a best featured area as a template and this selected template is located at $F_{x, y}$ coordinate point as shown in Fig. 3. It has $w$ pixel width and $h$ pixel height. As the next step, the selected template is saved as a sub image and this template image is used in subsequent template matching steps of the proposed method. All the template matching steps contain in the proposed method totally depend on selected template and its visual contents. Therefore, it is possible to select a template from the initial CCA frame which contains a good feature to match in subsequent frames. Based on the empirical results and strong observations of CCA, it is recommended to select a template around the catheter 
engaged area visualized in the first frame of selected CCA, as the best featured area to match during the matching step.

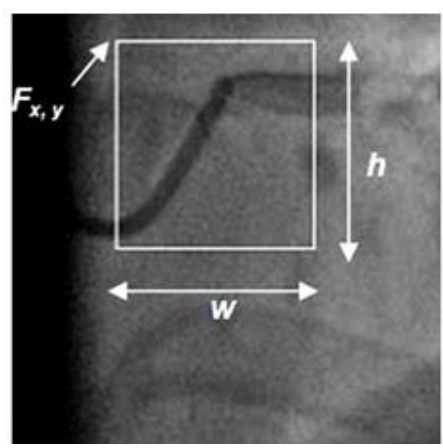

Fig. 3. Template selection

The second phase of the motion reduction process is the HRTM [13], [14]. It is an iterative process and runs up to the last frame of the CCA to be processed. The objective of the HRTM method is to efficiently compute the frame translation vectors of the CCA to be processed. That means the HRTM method finds the template image matching coordinates from the reference frame and returns the matched coordinate point as the output. Hence, in each recurring step, it requires the template image and the reference image which is the next frame of the CCA to be processed as inputs. During the iteration following algorithmic steps are executed;

\section{Step 1: Creating gaussian pyramids}

In HRTM, two Gaussian image pyramids are built recursively using both template and reference image. The next frame of the input CCA is selected as the reference image in each step. Depths of the both pyramids are set as two.

\section{Step 2: Apply template matching for the lowest level}

After creating two image pyramids the hierarchical template matching process begins from the lowest level (level depth 2) to highest level (level depth 0) of the pyramid iteratively. Correlation coefficient (CC) is selected as the similarity measure for the template matching step because it produces the lowest false matching occurrences in the validation process [14]. CC method applied in the template matching step is given in (3);

$$
R_{x, y}=\sum_{x^{\prime} y^{\prime}}\left[T^{\prime}\left(x^{\prime}, y^{\prime}\right) \cdot I^{\prime}\left(x+x^{\prime}, y+y^{\prime}\right)\right]^{2}
$$

where;

$$
\begin{aligned}
& T^{\prime}\left(x^{\prime}, y^{\prime}\right)=T\left(x^{\prime}, y^{\prime}\right)-T_{\text {mean }} \\
& I^{\prime}\left(x+x^{\prime}, y+y^{\prime}\right)=I^{\prime}\left(x+x^{\prime}, y+y^{\prime}\right)-I_{\text {mean }}
\end{aligned}
$$

$T$ and $I$ denote the template image and the reference image in the CCA respectively. $(x, y)$ denotes the coordinates of the matching region of the reference image and $\left(x^{\prime}, y^{\prime}\right)$ denotes the coordinates of the template. $T_{\text {mean }}$ gives the mean intensity of the template. In order to keep the computational output of the CC method, floating point image of size $(m-w+1)$ pixel width and $(n-h+1)$ pixel height is used as the result image which is denoted as $R . m$ and $n$ are the width and height of the matching region marked within the reference template where $m<$ template width and $n<$ template height. After that, following threshold function is applied on $R(x, y)$ image to obtain a binary image of it;

$$
\begin{gathered}
\text { if }(0.94<=R(x, y)<=1) \text { set } R(x, y) \text { as } 1 \\
\text { else set } R(x, y) \text { as } 0 .
\end{gathered}
$$

where $(x, y)$ denotes the spatial coordinates of the $R$;

This result image is used in the next levels of the Gaussian pyramid to effectively match and identify the template matching position from the reference image (see Fig. 4 (b)).

\section{Step 3: Apply template matching for the other levels}

The result image contents are copied in to the upper layer of the pyramid to effectively find the matched position. According to the CC method, the template matched position is located in the max regions $(R(x, y)=1)$ in the result image (see Fig. 4 (b)). Therefore, the template matching was done only for the predefined regions of interest $(R(x, y)=1$ regions $)$ in the upper levels of the image hierarchy and Fig. 4 (c) clearly depicts such detected contours of the result image shown in Fig. 4(b). After the template matching process, the threshold function is also applied for the result image generated in the upper levels of hierarchy as prescribed above. Finally, it returns the template matched position found in the reference frame when the template matching process reaches the highest level (level depth 0) in the hierarchy.

\section{Step 4: Compute the frame translation}

In order to construct the global motion eliminated frames, it is required to compute the frame translation vector between the matched template at the current frame and the selected template at the previous frame of two consecutive frames. Hence, the translation vector $M_{x, y}$ is given by;

$$
M_{x, y}=\left(C_{x}-F_{x}, C_{y}-F_{y}\right)
$$

where $C_{x}$ and $C_{y}$ denote the $x, y$ coordinates of the matched point obtained from $C_{x, y}$ point in the current frame and $F_{x}$ and $F_{y}$ denote the $x, y$ coordinates of the selected template point at $F_{x, y}$ coordinates in the previous frame (see Fig. 5). After determining the translation vector, the current frame must be aligned with the previous frame to produce the global motion eliminated frames.

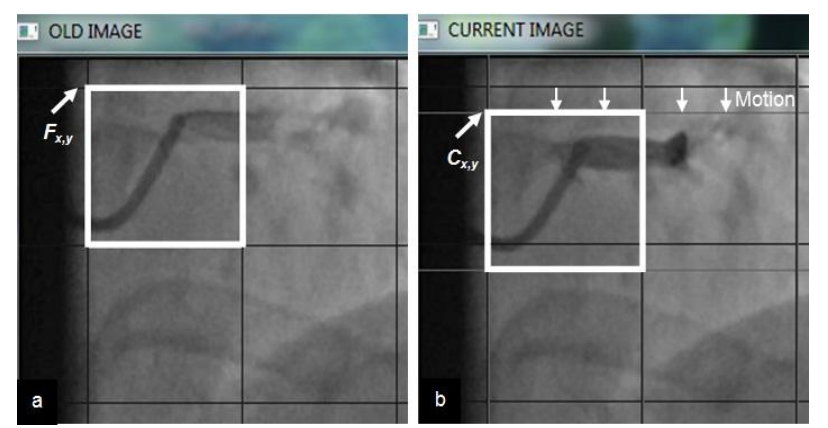

Fig. 5. Frame matching results; (a) template region at $F_{x, y}$ position; (b) matched template region at $C_{x, y}$ point. Arrow head represents the translation direction.

\section{Frame Alignment}

During this motion reduction process, the entire frame is translated into a certain calculated distance towards the direction determined by the translation vector. As a result of 
this translation a new frame is constructed. In this newly constructed frame, the matched template area (coordinates $C_{x, y}$ and dimension of $\left.(w \times h)\right)$ is positioned in the location $F_{x, y}$. After the first iteration, the aforementioned template selection step is done automatically using the newly constructed frame produced in the prescribed frame alignment step. This procedure repeats until the last frame of the input CCA. The final outcome of this global motion reduction iterative process is a set of global motion eliminated Angiogram images of the input preprocessed CCA.

\section{Creating Motion Reduction CCA}

Motion eliminated video creation process starts after the frame alignment process. It creates a global motion reduced video using the global motion eliminated images of the preprocessed CCA which are produced by the previous steps. The frame rate and size of the frames of the motion eliminated video are set the same with the preprocessed video frame rate and size to maintain the Angiography standards.

\section{RESUlts}

In order to validate this proposed HRTM method, we have run the developed algorithm of the proposed method using the data set of twenty randomly selected angiogram cases. It consists of 119 CCAs which were taken under the different views. Template selection for each execution was done randomly. Hence, we are able to analyze 2707 total frames to validate the accuracy of this proposed method. Some important information about the test data set is included in Table I.

We have tested the proposed algorithm for five different similarity measures defined in OpenCV vision library to select the best matched similarity measure for the template matching operation. Table II clearly depicts the results of this validation method. A set of visually aligned frames of the CCA corresponding to the frames shown in Fig. 1 is given in Fig. 6 to visually compare the results obtained from this proposed frame alignment method.
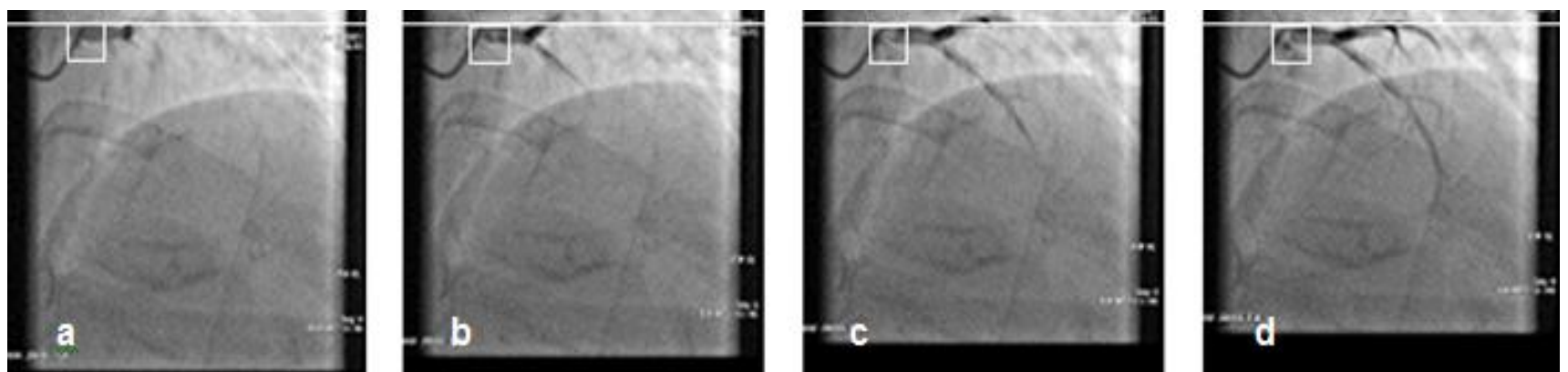

Fig. 6. Motion compensated visually aligned frames of a CCA. Catheter engaged area is marked using whit colored square and placement of it is stable in the four consecutive frames shown in a,b,c and $\mathrm{d}$.
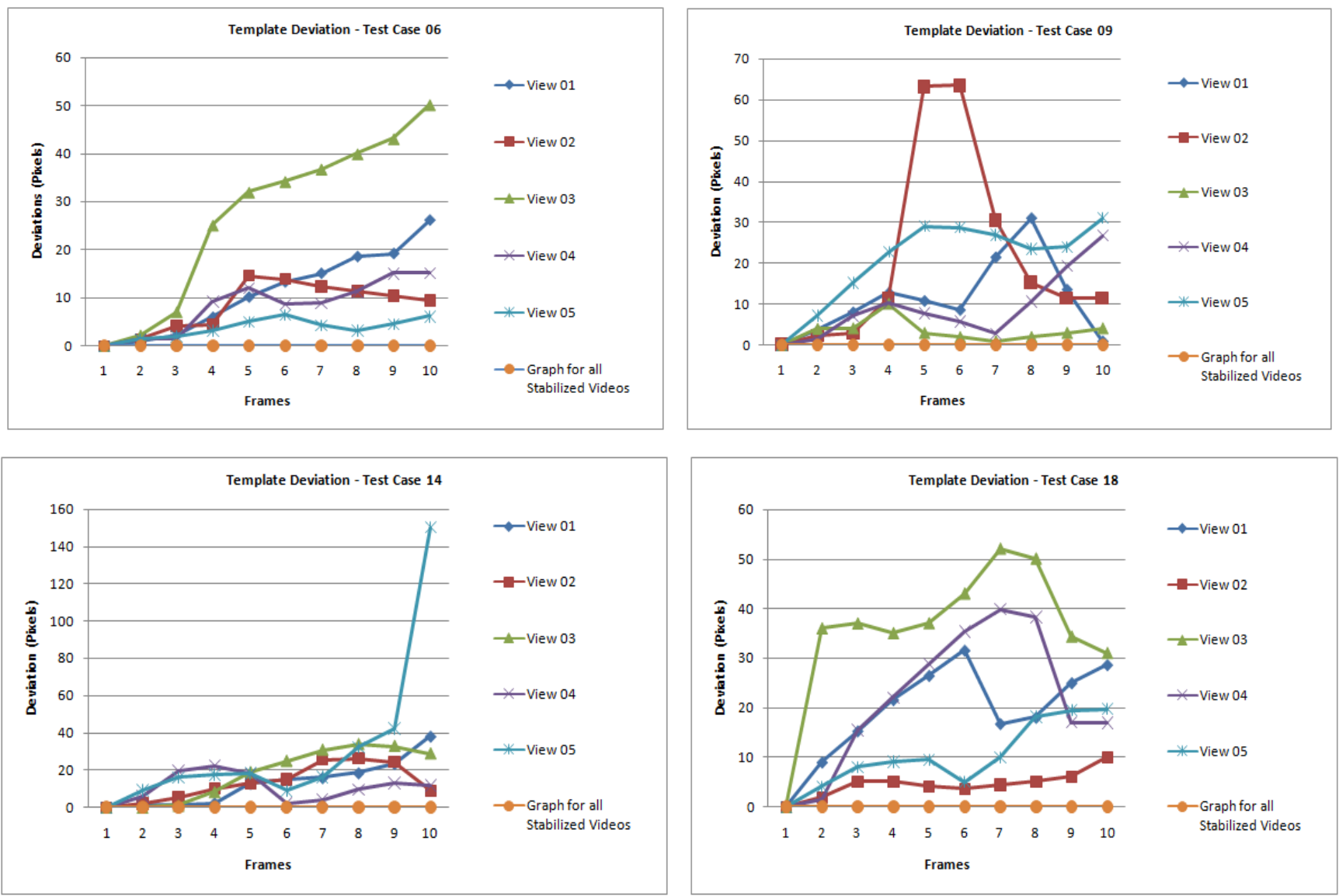

Fig. 7. Distance deviation of the templates from the initial template of four cases. 
TABLE I: SUMMARY OF CCAS OF THE TEST DATA SET

\begin{tabular}{llll}
\hline \hline CCA No & $\begin{array}{l}\text { Views } \\
\text { Per CCA }\end{array}$ & $\begin{array}{l}\text { Total frames } \\
\text { for matching }\end{array}$ & $\begin{array}{l}\text { True Positive } \\
\text { matching percentage }\end{array}$ \\
\hline 1 & 7 & 161 & 93.17 \\
2 & 7 & 146 & 94.52 \\
3 & 3 & 58 & 98.28 \\
4 & 6 & 98 & 96.94 \\
5 & 3 & 53 & 96.23 \\
6 & 5 & 70 & 98.57 \\
7 & 10 & 187 & 96.79 \\
8 & 8 & 115 & 97.39 \\
9 & 5 & 103 & 94.17 \\
10 & 10 & 229 & 94.32 \\
11 & 8 & 267 & 98.13 \\
12 & 6 & 198 & 95.45 \\
13 & 4 & 123 & 95.12 \\
14 & 5 & 125 & 97.60 \\
15 & 6 & 128 & 94.53 \\
16 & 7 & 201 & 97.51 \\
17 & 4 & 94 & 98.94 \\
18 & 5 & 128 & 94.53 \\
19 & 5 & 105 & 98.10 \\
20 & 5 & & 93.22 \\
\hline \hline
\end{tabular}

The template distance deviation between the initial template and the matched template obtained in each iterative step of this motion reduction method was calculated and plotted for both preprocessed CCA in the data set (Table I) and global motion eliminated version of those preprocessed CCAs. Fig. 7 depicts sample template deviation graphs of five views of four selected CCA cases extracted from the test dataset for further clarification. Moreover, it contains the common graph plotted to represent the distance deviation of global motion eliminated versions of input CCAs.

\section{DISCUSSION}

According to the results of this proposed methodology, it is apparent that motion reduction in CCA improves visual alignment of vessel structures and arterial flow between multiple frames. This can be clearly identified by looking at the catheter engaged area of both original and global motion eliminated frame sequences shown in Fig. 1 and Fig. 6. The catheter engaged area is marked as a white colored square in both image sets. According to the Fig. 6, motion reduced frames depict catheter engaged area in a fixed location. This happens as a result of reconstructing the frame by reducing the global motion. Further, this frame reconstruction is done based on the calculated translation vector.

The template matching is also done using two image pyramids created for both template image and the reference frame to be matched. These image pyramids consist of three different levels (depth of both pyramids is taken as 2) with different dimensions of template and reference images. The exact template matching started from the lower level of the pyramid (depth $=0$ ) and the results of it return to the upper levels to find the best matched coordinates from the reference image. Template matching was applied only for detected contours in upper levels of the image pyramid and as a result of that, this proposed matching method improves the efficiency of template matching process by reducing unnecessary comparisons required to find the marked template image.

$\mathrm{CC}$ was selected as the similarity measure in the template matching steps because it is revealed that $\mathrm{CC}$ produces the highest percentage of true matches during the validation process (Table II). Further, CC method match a template relative to its mean against the image relative to its mean [14].

\begin{tabular}{ll} 
TABLE II: MATCHING STRENGTH OF VARIOUS SIMILARITY MEASURES \\
\hline \hline Technique & True Positive Matching \% \\
\hline Square Difference & 31.35 \\
Square Difference Normalize & 31.35 \\
Correlation Normalized & 84.33 \\
Correlation Coefficient & 94.05 \\
Normalize Correlation Coefficient & 31.35 \\
\hline \hline
\end{tabular}

Graphs shown in Fig. 7 clarify the global motion reduction capability of this proposed method. According to that, for all $\mathrm{CCA}$, the template deviation is varying with respect to the frames due to the effect of global motion. The experimental results have shown that the distance deviation was computed as zero for all global motion eliminated CCAs of input dataset. Hence, the created graphs for global motion eliminated versions are same for all CCAs in the Table I. Template deviation becomes 0 means; the matched template points of global motion eliminated frames are found from the same location where the first selected template was located. This happens due to the reduction of global motion artifact from each and every processed frame of input CCA.

A recent research publication has pointed out that, the motion of anatomy is complex and cannot be accurately corrected by estimating models with low degrees of freedom such as rigid or affine transformation [15]. Our study also aligns with this fact. However, it is not a big issue in our study, because our major target is improving the visual alignment of the vessel structure among the CCA's frame sequence. The past research studies have explained the application of image registration techniques for motion correction in Digital Subtraction Angiograms [6]-[8]. When compared to the image registration algorithms, template matching is simpler and it is not based on a complex mathematical model. Moreover, the image registration techniques contain a level of uncertainty if the registering images have any spatio-temporal differences [16]. CCAs contain some spatio-temporal differences from frame to frame due to the natural transformations of recorded vessel structures. Hence, CCAs can contain some difficulties to apply image registration for reducing the global motion artifacts and as a consequence, the image registration techniques do not provide reliable results always for CCAs.

\section{CONCLUSION AND FUTURE WORK}

This paper describes an efficient approach in global motion reduction technique to obtain visual alignment of CCAs. Compared to the other motion reduction methods, the proposed method can be directly applied to the direct CCAs and it follows a hierarchical template matching based technique to reduce the motion disturbances. Experimental results of this proposed method have clearly shown how to 
minimize the long visual gaps of the vessel structure among the frames using template matching technique. Hence, it is possible to improve the visual alignment among the frames of CCA and as a result of that, we can obtain a smooth flow of contrast agent within the vessel structure starting from a predefined location.

As future work, this proposed technique can be further, enhanced by using different Angiogram views. It is possible to further improve the Angiography technique to detect and quantify the stenosis severity based on functional information such as flow rate and flow velocity using the results obtained from the proposed HRTM method.

\section{ACKNOWLEDGMENT}

The authors thank the staff of the Cardiology Unit of the National Hospital Sri Lanka and the Cath Lab Staff members of the Asiri Surgical Hospitals.

\section{REFERENCES}

[1] J. T. Wong, H. Le, W. M. Suh, D. A. Chalyan, T. Mehraien, M. J. Kern, G. S. Kassab, and S. Molloi, "Quantification of fractional flow reserve based on angiographic image data," Int. J. Cardiovasc. Imaging, vol. 28, no. 1, pp. 13-22, Jan. 2012.

[2] P. A. L. Tonino, W. F. Fearon, B. De Bruyne, K. G. Oldroyd, M. A Leesar, P. N. Ver Lee, P. A. MacCarthy, M. van't Veer, and N. H. J. Pijls, "Angiographic versus functional severity of coronary artery stenoses in the FAME study: Fractional flow reserve versus angiography in multivessel evaluation," J. Am. Coll. Cardiol., vol. 55 , no. 25, pp. 2816-2821, Jun. 2010.

[3] M. T. Dehkordi, S. Sadri, and A. Doosthoseini, "A review of coronary vessel segmentation algorithms," J. Med. Signals Sensors, vol. 1, no. 1 , pp. 49-54, Jan. 2011

[4] A. S. Roy, M. R. Back, S. F. Khoury, E. W. Schneeberger, L. H. Back, V. V. Velury, R. W. Millard, and R. K. Banerjee, "Functional and Anatomical Diagnosis of Coronary Artery Stenoses," J. Surg. Res., vol. 150 , no. 1, pp. 24-33, Nov. 2008.

[5] A. Kusumawardhani, T. Mengko, I. Fahri, S. Soerianata, D. Firman, and H. Zakaria, "Coronary angiogram stabilization for QuBE values calculation using SIFT method," in Proc. 2011 2nd International Conference on Instrumentation, Communications, Information Technology, and Biomedical Engineering (ICICI-BME), 2011, pp. 111-116.

[6] M. S. D. Kumar, D. Shen, L. Wei, R. Turlapthi, and J. S. Suri, "Motion correction strategies for interventional angiography images: A comparative approach," in Proc. IEEE International Conference on Image Processing, 2007, vol. 1, pp. I-497-I-500.

[7] E. H. W. Meijering, W. J. Niessen, J. Bakker, A. J. van der Molen, G. A. P. de Kort, R. T. H. Lo, W. P. T. M. Mali, and M. A. Viergever, "Reduction of patient motion artifacts in digital subtraction angiography: Evaluation of a fast and fully automatic technique," Radiology, vol. 219, no. 1, pp. 288-293, Apr. 2001.

[8] C.-C. Ko, C.-W. Mao, and Y.-N. Sun, "Multiresolution registration of coronary artery image sequences," Int. J. Med. Inf., vol. 44, no. 2, pp. 93-104, Apr. 1997.

[9] S. Klein, M. Staring, K. Murphy, M. A. Viergever, and J. P. W. Pluim, "Elastix: A toolbox for intensity-based medical image registration," IEEE Trans. Med. Imaging, vol. 29, no. 1, pp. 196-205, Jan. 2010

[10] J. Meunier, M. G. Bourassa, M. Bertrand, M. Verreault, and G. E. Mailloux, "Regional epicardial dynamics computed from coronary cineangiograms," in Proc. Computers in Cardiology, 1989, pp. $307-310$.

[11] S. Zheng and D. Weirong, "Analysis of coronary arterial dynamics from x-ray angiographic sequences," in Proc. Second International Symposium on Computational Intelligence and Design, 2009, vol. 1 , pp. $201-204$.
[12] M. A. U. Khan, M. K. Khan, and M. A. Khan, "Coronary angiogram image enhancement using decimation-free directional filter banks," in Proc. IEEE International Conference on Acoustics, Speech, and Signal Processing, 2004, vol. 5.

[13] R. Brunelli, "Computational aspects of template matching," Template Matching Techniques in Computer Vision: Theory and Practice, John Wiley \& Sons, pp. 121-122, 2009.

[14] G. Bradski and A. Kaehler, "Template matching," Learning OpenCV: Computer Vision with the OpenCV Library, 1st ed. O'Reilly Media, 2008, pp. 214-219.

[15] M. S. D. Kumar, L. Wei, R. Turlapthi, and J. S. Suri, "DSA Image enhancement via multi-resolution motion correction for interventional procedures: A robust strategy," in Proc. the fifth IASTED International Conference: Biomedical Engineering, Anaheim, CA, USA, 2007, pp 330-335.

[16] K. M. Simonson, S. M. Drescher, and F. R. Tanner, "A statistics-based approach to binary image registration with uncertainty analysis," IEEE Trans. Pattern Anal. Mach. Intell., vol. 29, no. 1, pp. 112-125, 2007.

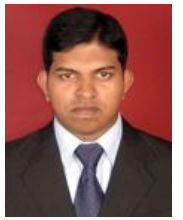

K. A. S. H. Kulathilake received the B.Sc. special degree in information technology from Sri Lanka Institute of Information Technology, Sri Lanka in 2007 and master of computer science degree from University of Colombo, Sri Lanka in 2011. He is an M.Phil candidate in medical image processing at the University of Moratuwa, Sri Lanka.

$\mathrm{He}$ is a probationary lecturer at the Rajarata University of Sri Lanka attached to the Department of Physical Sciences since 2009.

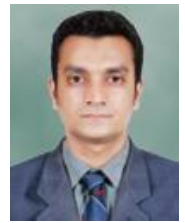

L. Ranathunga received the B.Sc. special degree in computer science from University of Peradeniya, Sri Lanka in 1999 and M.Sc. in computer science degree from University of Colombo, Sri Lanka in 2005. He received $\mathrm{PhD}$ from University of Malaya, Malaysia in 2011.

$\mathrm{He}$ is a senior lecturer at the University of Moratuwa, Sri Lanka attached to the Department of Information Technology. He has presented a number of papers in International Conferences and also attended Workshops and Seminars. He has also published several articles in peer reviewed indexed journals. His area of research is multimedia and allied technologies

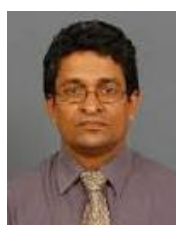

G. R. Constantine received the MBBS degree from University of Jaffna, Sri Lanka in 1991 and MD in cardiology from University of Colombo, Sri Lanka in 1999.

$\mathrm{He}$ is a senior lecturer at the University of Colombo, Sri Lanka attached to the Department of Clinical Medicine. He has presented a number of papers in National and International Conferences and also attended Workshops and Seminars. He has also published several articles in peer reviewed indexed journals. His areas of research are cardiovascular effects of renal failure, role of echocardiogram in haemodynamically significant pulmonary embolism and pathophysiology of resistant hypertension multimedia and allied technologies.

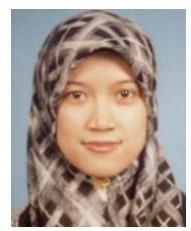

N. A. Abdullah received the B.Sc. degree in computer science from University of Malaya, Malaysia and M.Sc. in computer science degree from University of Westminster. She received PhD in computer science from University of Southampton, UK.

She is an associate professor at the University of Malaya, Malaysia attached to the Department of Computer Systems and Technology. She has presented a number of papers in International Conferences and also published several articles in peer reviewed indexed journals. She is doing research on areas such as; Multimedia, Algorithms \& Techniques and E-Leaening.

Professor Aniza is an ordinary member of Association for Computing Machinery and IEEE since 2013. 